Pathologe 2008 $29: 199-204$

DOI 10.1007/s00292-008-0997-z

Online publiziert: 9. April 2008

(c) Springer Medizin Verlag 2008

\author{
G. Jundt ${ }^{1}$ P.A. Reichart ${ }^{2}$ \\ ${ }^{1}$ Institut für Pathologie, DÖSAK-Zentralregister am Knochentumor- \\ Referenzzentrum, Universitätsspital Basel, Schweiz \\ ${ }^{2}$ Abteilung Oralchirurgie und Zahnärztliche Röntgenologie, \\ Charité - Universitätsmedizin Berlin
}

\title{
Benigne odontogene ektomesenchymale Tumoren
}

\section{Klassifikation}

Die Gruppe der odontogenen ektomesenchymalen Tumoren, die per definitionem keine epitheliale Tumorkomponente besitzen, sondern allenfalls eingeschlossenes odontogenes Epithel als Hinweis auf ihre Herkunft aufweisen, besteht aus 3 Läsionen (• Tab. 1):

- odontogenes Fibrom,

- odontogenes Myxom,

- Zementoblastom.

Gegenüber der Klassifikation odontogener Tumoren von 1992 haben sich in der neuen Klassifikation von 2005 keine wesentlichen Neuerungen ergeben. Die Terminologie der odontogenen Fibrome wurde präziser gefasst und das Wort „benigne" aus der Bezeichnung für das Zementoblastom gestrichen $[2,16]$. Die Bedeutung immunhistochemischer Untersuchungen ist marginal und hilft allenfalls bei der Identifizierung odontogenen

Tab. 1 Benigne odontogene ektomesenchymale Tumoren mit oder ohne odontogenes Epithel

\begin{tabular}{ll} 
Klassifikation & ICD-O Code \\
$\begin{array}{l}\text { Odontogenes Fibrom } \\
\text { Epithelreich } \\
\text { Epithelarm }\end{array}$ & $9321 / 0$ \\
\hline $\begin{array}{l}\text { Odontogenes Myxom } \\
\text { Zementoblastom }\end{array}$ & $9320 / 0$ \\
\hline
\end{tabular}

Epithels. Dies kann jedoch die Diagnose und Differenzialdiagnose wesentlich erleichtern.

\section{Odontogenes Fibrom}

Das odontogene Fibrom (OF) ist ein sehr seltener, nichtinvasiver odontogener Tumor unklarer Ätiologie, der aus einem reifen, bindegewebigen Stroma besteht und kleine Stränge inaktiven odontogenen Epithels enthält [20]. Je nach Menge des Epithels können ein epithelarmer und ein epithelreicher Subtyp unterschieden werden. Nach Gardner [10] leitet sich der epithelarme Subtyp vom Zahnfollikel und der epithelreiche vom periodontalen Ligament ab [10]. Bisher sind etwa 7o Fälle publiziert worden [7]. Da die diagnostischen Kriterien jedoch nicht einheitlich waren und die histologische Dokumentation der Fälle oft unzureichend ist, lassen sich selbst relative Häufigkeitsangaben nur sehr schwer ermitteln. Der Tumor kommt in allen Altersgruppen bei einem Durchschnittsalter von etwa 40 Jahren vor. Frauen sind etwa 3-mal häufiger betroffen als Männer [20].

Hauptsitz ist die Prämolarenregion der Mandibula. Neben den hier diskutierten intraossären oder zentralen $\mathrm{OF}$ kommen auch extraossäre (periphere) Läsionen vor $[11,12]$.

Die klinische Symptomatik ist oft gering. Manchmal machen die Läsionen über eine langsam zunehmende Schwellung auf sich aufmerksam.

Radiologisch sieht man bei kleinen Läsionen gut begrenzte, unilokuläre Osteolysen, die in Kontakt zu Zahnwurzeln stehen können. Größere Läsionen sind multilokulär gestaltet und zeigen einen oft bogenförmig konturierten, partiell sklerotischen Randsaum ( $\bullet$ Abb. 1 a). In wenigen Fällen können Kalzifikationen beobachtet werden, ebenso Wurzelspitzenresorptionen und Verlagerungen von Zähnen [20].

Makroskopisch ist das Fibrom weiß und solide. Histologisch kann zwischen einem epithelarmen (früher: einfachen) und einem epithelreichen (früher: WHOTyp) OF unterschieden werden. Die epithelarme Variante besitzt ein lockeres, fibromyxoides Stroma mit spindelig- bis sternförmigen Fibroblasten und zarten bis kräftigen Kollagenfasern (• Abb. 1 d). Sie enthält außerdem wenige odontogene Epithelien, gelegentlich spärliche Verkalkungen und besitzt große Ähnlichkeit mit Strukturen des Zahnfollikels. Der epithelreiche Typ zeigt abwechselnd zellreiche, stärker kollagenisierte und lockere, vaskularisierte Areale und bereits bei kleiner Vergrößerung erkennbare Gruppen odontogener epithelialer Zellnester, die gelegentlich dominieren können

Widmung. Herrn Professor Dr. med. Wolfgang Remagen zu seinem 80. Geburtstag gewidmet 

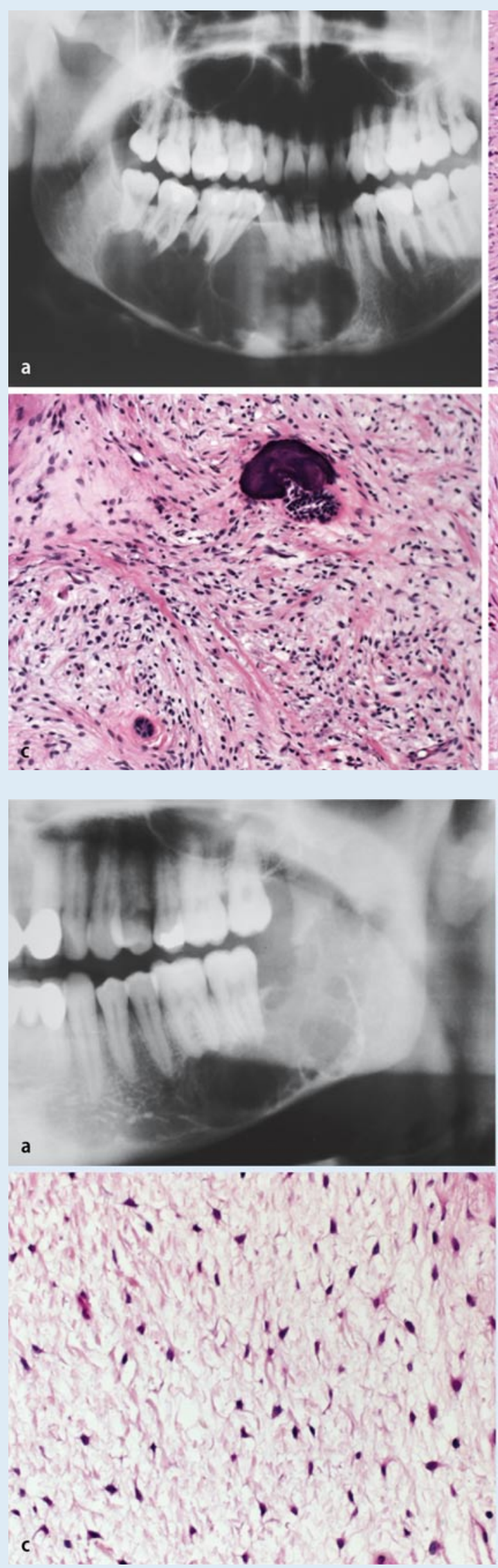
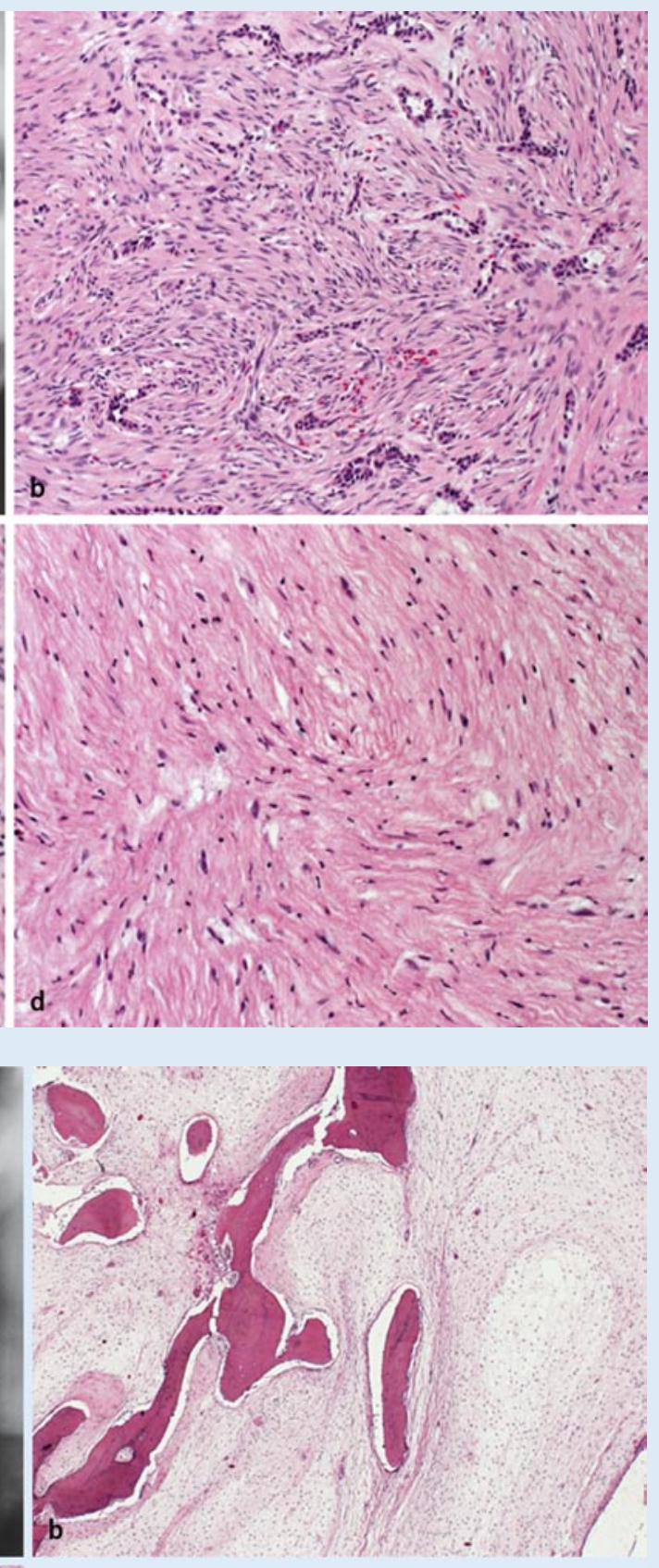

Abb. $2<$ Odontogenes Myxom. a Ausgedehnte, teilweise fein trabekulierte Osteolyse des linken horizontalen Unterkieferastes mit Übergreifen auf den aufsteigenden Ast. Im aufsteigenden Ast ist ein wabenartiges Destruktionsmuster erkennbar. b Infiltration des ortsständigen Knochens durch ein lockeres, relativ zellarmes, mukopolysaccharidreiches Tumorgewebe (HE-Färbung, Vergr. 1:50). c Die Tumorzellgrenzen sind schlecht erkennbar. Die Kerne sind teils zipflig ausgezogen, teils triangulär und chromatindicht (HE-Färbung, Vergr. 1:400) 
(- Abb. 1 b, c). Diese Epithelien sind inaktiv und zeigen keine periphere Palisadierung im Unterschied zum Ameloblastom. Daneben sieht man Hartsubstanzablagerungen (dysplastisches Dentin, Zement- oder Knochenbildung), die gelegentlich sogar als intraläsionale Verkalkungen radiologisch sichtbar werden können (• Abb.1 a). Eine granularzellige Variante wurde ebenfalls beschrieben [22].

Sowohl das zentrale als auch das periphere odontogene Fibrom zeigen nur eine geringe Infiltrationstendenz. Therapeutisch ist deshalb die Enukleation der oft gut begrenzten Läsionen meist ausreichend. Rezidive werden nur selten beobachtet.

Differenzialdiagnostisch muss das epithelarme odontogene Fibrom von einem desmoplastischen Fibrom abgegrenzt werden (kein eingeschlossenes odontogenes Epithel), was gelegentlich trotz Aufarbeitung in Serienschnitten und immunhistochemischen Nachweisversuchen zytokeratinpositiven odontogenen Epithels schwierig bis unmöglich sein kann. Der epithelreiche Typ kann Ähnlichkeit zu einem verkalkenden epithelialen odontogenen Tumor aufweisen. Im Gegensatz zum OF enthält der CEOT immer amyloidartiges Material, sodass eine Unterscheidung mit der Kongo-Rot-Färbung gelingt. Außerdem besitzen die Plattenepithelien des CEOT ein wesentlich breiteres Zytoplasma und sind oft durch Interzellularbrücken miteinander verbunden. Beide Varianten können mit Fragmenten des Zahnfollikels verwechselt werden, wenn dem Röntgenbild (perikoronale Aufhellung im Falle eines hyperplastischen Follikels) keine Beachtung geschenkt wird.

\section{Odontogenes Myxom (Myxofibrom)}

Das odontogene Myxom (OM) ist ein intraossärer, praktisch nur im Kieferbereich vorkommender Tumor, der aus sternförmigen, spindeligen Zellen besteht, die in eine myxoide extrazelluläre Matrix eingebettet sind [5].

Der Tumor ist relativ selten. Seine relative Häufigkeit beträgt nach den Angaben verschiedener Studien aus Asien, Afrika, Europa, Nord- und Südamerika zwischen

Pathologe 2008 $\cdot 29: 199-204 \quad$ DOI 10.1007/s00292-008-0997-z

(c) Springer Medizin Verlag 2008

\section{G. Jundt $\cdot$ P.A. Reichart \\ Benigne odontogene ektomesenchymale Tumoren}

\section{Zusammenfassung}

Die Gruppe der odontogenen ektomesenchymalen Tumoren wird von 3 Läsionen gebildet, dem odontogenen Fibrom (epithelarm und epithelreich), dem odontogenen Myxom und dem Zementoblastom. Während odontogene Fibrome und Zementoblastome sehr selten sind, ist das odontogene Myxom nach dem keratozystischen odontogenen Tumor, den Odontomen und dem Ameloblastom der vierthäufigste odontogene Tumor. Die Diagnose des Zementoblastoms ergibt sich aus seiner engen Assoziation mit einer Zahnwurzel. Die Abgrenzung odontogener Fibrome und Myxome kann jedoch schwierig werden, da sie histologisch große Ähnlichkeiten mit normalen Zahnstrukturen (Zahnpapille, Zahnfollikel) besitzen, wobei die letzteren häufig als odontogene Tumoren, besonders Myxome, fehlinterpretiert werden, wenn das Röntgenbild (umschriebene Osteolyse mit einem retinierten Zahn) nicht beachtet wird und andere Hinweise (oberflächliche Reste des Schmelzepithels, Dentinfragmente) nicht gesucht werden. Während odontogene Fibrome kaum rezidivieren und lokal exzidiert werden können, zeigen das Zementoblastom und insbesondere das odontogene Myxom eine ausgeprägte Rezidivneigung, sodass besonders bei Myxomen im Oberkiefer primäre Resektionen erforderlich werden, um ein Übergreifen auf Orbita oder Schädelbasis zu verhindern.

\section{Schlüsselwörter}

Odontogenes Fibrom · Odontogenes Myxom · Zementoblastom · Zahnfollikel . Zahnpapille

\section{Benign odontogenic ectomesenchymal tumors}

\section{Abstract}

The group of odontogenic ectomesenchymal tumors consists of odontogenic fibroma (epithelium-rich and epithelium-poor types), odontogenic myxoma, and cementoblastoma. Whereas odontogenic fibromas and cementoblastomas are very rare lesions, odontogenic myxoma is the fourth common odontogenic tumor, preceded only by keratocystic odontogenic tumor, the odontomas, and ameloblastoma. The diagnosis of cementoblastoma rests on its connection to the root of a tooth. The differentiation of odontogenic fibroma and myxoma from other lesions, especially from normal structures such as dental follicles and papillae, may be challenging if the X-ray appearance (localized osteolysis containing a tooth) is not appreciated and subtle histological clues (remainders of inner enamel epithelium at the surface of the lesion, dentin fragments) are not properly recognized. While odontogenic fibromas have almost no tendency for recurrence and are treated by enucleation or local excision, cementoblastomas and especially odontogenic myxomas have a high percentage of recurrence if intralesional procedures are applied. Hence, complete resection with free margins is recommended-at least for larger odontogenic myxomas and, especially, lesions in the maxilla-to prevent further extension to the orbita or base of the skull.

\section{Keywords}

Odontogenic fibroma - Odontogenic myxoma $\cdot$ Cementoblastoma $\cdot$ Dental follicle $\cdot$ Dental papilla 


\section{Schwerpunkt: Zahnpathologie}

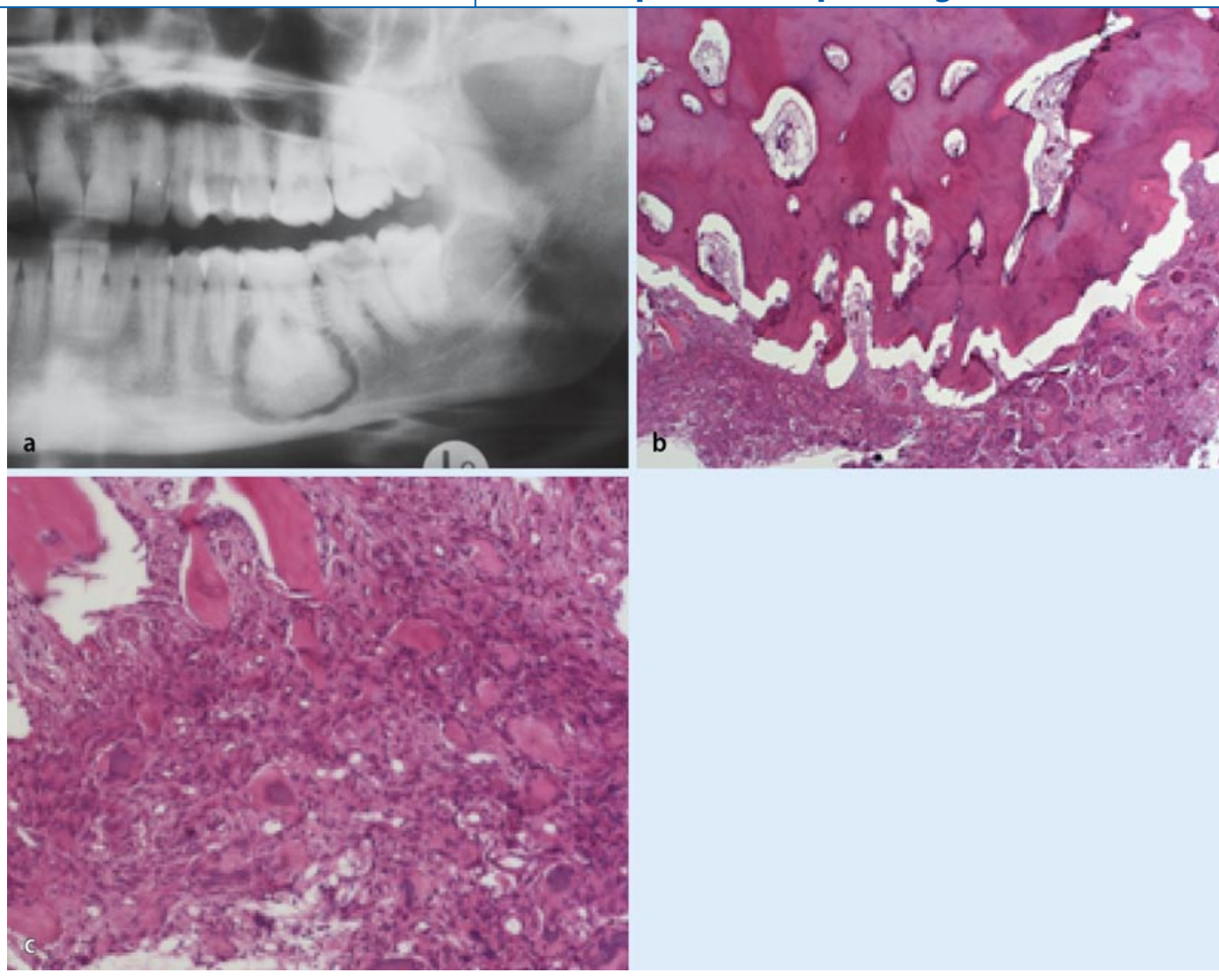

Abb. $3<$ Zementoblastom

a Annähernd rundliche Verschattungszone in engem

Kontakt zu den Wurzeln des Zahnes 36, die von der Verschattung nicht mehr zu trennen sind. Die Läsion wird peripher von einem scharf begrenzten Aufhellungssaum umgeben. Röntgenbild nahezu pathognomonisch für Zementoblastom.

b In den oberen 2/3 zellarme Zementmassen, an deren Oberflächen keine aktiven Zellelemente sichtbar sind. Die das untere Drittel einnehmende periphere Zone entspricht der Aufhellungszone im Röntgenbild und enthält neben zellulären Elementen zahlreiche zementikelartige Hartsubstanzablagerungen (HE-Färbung, Vergr. 1:50). c Ausschnitt aus der peripheren Aufhellungszone: links oben Ausläufer säulenartig angeordneter Zementmassen, daran anschließend Stromazellen, zahlreiche Zementoblasten sowie zementikelartige Hartsubstanzneubildungen, einzelne Riesenzellen und Gefäße (HE-Färbung, Vergr. 1:200)

$4,7-17,7 \%$ und ist damit nach dem keratozystischen odontogenen Tumor, den Odontomen und dem Ameloblastom der vierthäufigste odontogene Tumor [13]. Es kann in allen Lebensaltern vorkommen, hauptsächlich in der 2. bis 4. Lebensdekade mit einem Durchschnittsalter von etwa 30 Jahren und einem leichten Überwiegen des weiblichen Geschlechts [5]. Kinder sind sehr selten betroffen [14]. Etwa 2/3 der Fälle sind im Unterkiefer lokalisiert, besonders in der Molarregion.

Während kleine OM meist zufällig entdeckt werden, führen größere Läsionen zu einer Expansion und Auftreibung des Kiefers. Schmerzen und Parästhesien sind selten. Oberkiefermyxome können zu sinonasalen Obliterationen unter dem Bild einer Polyposis nasi führen [5].

Die Ätiologie ist unklar. Während einige Autoren eine Beziehung des Tumors zur Zahnpapille oder dem Zahnfollikel annehmen, sehen andere überhaupt keinen Zusammenhang mit odontogenen Strukturen, sondern eher eine Beziehung zu Myofibroblasten und Fibroblasten, zumal eingeschlossenes odontogenes Epithel eher die Ausnahme als die Regel ist [21].
Kürzlich konnte jedoch in Myxomen mit und ohne eingeschlossenes odontogenes Epithel das Intermediärfilament Nestin nachgewiesen werden [9], das zunächst in embryonalen Stammzellen entdeckt wurde, aber auch im Ektomesenchym des sich entwickelnden Zahns, der Zahnleiste und im Schmelzorgan gefunden werden konnte $[18,26]$. Dies kann als deutlicher Hinweis auf eine Abstammung des OM vom Ektomesenchym angesehen werden. Ob sich der immunhistochemische NestinNachweis auch als differenzialdiagnostisches Kriterium durchsetzen wird, muss noch abgewartet werden.

Radiologisch sieht man unilokuläre oder (häufiger) multilokuläre, gelegentlich seifenblasenartige Osteolysen mit meist scharfer Begrenzung und Verdrängung der Zähne, manchmal auch in Verbindung mit einem retinierten Zahn (- Abb. 2 a). Selten zeigen sie eine charakteristische, feine interne, rechtwinklig angeordnete Trabekulierung, die an das Saitenmuster eines Tennisschlägers erinnern, und die sich mit Spezialtechniken wie Cone-Beam-CT besser darstellen lassen [1]. Wurzelverlagerungen und Wurzel- spitzenresorptionen können beobachtet werden. Eine sonnenstrahlenartige („sunray appearance") Periostreaktion, wie sie im Kiefer bei Osteosarkomen, Metastasen und gelegentlich auch bei der Osteomyelitis vorkommen kann, wurde ebenfalls beschrieben [6].

Makroskopisch ist der Tumor glänzend, grau-weiß, gelegentlich durchscheinend und bietet eine gelatinöse bis feste Konsistenz, abhängig von seinem Gehalt an Kollagenfasern. Histologisch sieht man relativ gleichmäßig verteilte, spindelige Zellen, die in eine myxomatöse Matrix eingebettet sind. Die Zellen besitzen kleine, rundliche bis ovale, gelegentlich triangular wirkende, chromatindichte Kerne und zipflig ausgezogene, blass eosinophile Zytoplasmafortsätze (• Abb. 2 a, c). Mitosen sind sehr selten, eine mäßige Zellpleomorphie und einzelne doppelkernige Zellen kommen aber vor. Eingeschlossenes odontogenes Epithel ist die Ausnahme. Der Tumor durchsetzt diffus infiltrierend den Knochen, ohne dass eine klare Begrenzung zur Umgebung erkennbar wird ( $\bullet$ Abb. 2 b). Zellreichere Varianten mit größeren, atypischen Ker- 
nen, in Einzelfällen auch mit einem sarkomatösen Aspekt und infiltrativ-destruktivem Wachstum sind ebenfalls beschrieben [17] und haben in einem Fall über eine intrakranielle Ausbreitung zum Tode des Patienten geführt. Dieser Fall zeigte auch komplexe chromosomale Aberrationen [19]. Da Metastasen bis jetzt jedoch noch nicht beschrieben wurden, sollte in diesen - sehr seltenen - Fällen nicht von einer Transformation in ein Sarkom gesprochen werden.

OM haben keine Begrenzung und besitzen ein infiltratives Wachstumsverhalten. Deshalb werden nach Kürettage in bis zu 25\% Rezidive beobachtet, die meist innerhalb von 2 Jahren auftreten [5]. Besonders bei maxillären $\mathrm{OM}$ wird deshalb eine komplette Resektion empfohlen, da Rezidive in dieser Region bei nicht sicher erkennbaren Tumorgrenzen kaum beherrschbar sind [21].

Die wichtigste Differenzialdiagnose ist die Abgrenzung eines OM von normalen Zahnstrukturen, besonders von der Zahnpapille. Bereits die Beachtung des Alters (selten bei Kindern) und des Röntgenbilds ist hier sehr hilfreich, da Myxome in der Regel keine retinierten Zähne enthalten. Deshalb sollte man bei einem retinierten Zahn extrem zurückhaltend mit der Diagnose OM (oder OF) sein, wenn das Röntgenbild keine eindeutige tumorbedingte Osteolyse aufweist. Außerdem zeigen Papillenfragmente fast immer zumindest kleine Reste benachbarter odon- togener Strukturen wie zytokeratinpositive Zellgruppen des inneren Schmelzepithels oder Dentinfragmente an ihrer Oberfläche [15, 23].

\section{Zementoblastom}

Das Zementoblastom (ZB) ist ein odontogener Tumor mit ausgeprägten Zementablagerungen, die sich in unmittelbarem Kontakt zu einer Zahnwurzel befinden [25]. Die Läsion tritt selten vor dem 10. und nach dem 7o. Lebensjahr auf und zeigt einen Gipfel im 2. und 3. Lebensjahrzehnt bei einem Durchschnittsalter von 21,3 Jahren. 70\% aller ZB werden vor dem 30. Lebensjahr beobachtete. Das männliche Geschlecht ist in etwa 58\% der Fälle betroffen [4, 21].

Die Ätiologie ist unklar. Argumente für einen eigenständigen, sich von Zementoblasten bzw. von ektomesenchymalen periodontalen Zellen ableitenden Tumor sind seine Entwicklung unterhalb des periodontalen Ligaments außerhalb des Knochens und sein unmittelbarer Kontakt zur Zementschicht, die die Zahnwurzel überzieht [4].

Etwa 80\% der Fälle treten im Unterkiefer auf und sind bis auf wenige Ausnahmen mit der Wurzel eines Molaren oder Prämolaren verbunden. Der Befall eines Milchzahns ist eine Rarität. Schmerzen treten häufiger als bei anderen odontogenen Tumoren etwa in 60\% der Fälle auf. Da das ZB ein kontinuierlich wach- sender Tumor ist, kommt es zu einer Auftreibung des Kiefers. Die in die Läsion einbezogenen Zähne bleiben fast immer vital [21].

Radiologisch sieht man in frühen Stadien zunächst eine scharf begrenzte, im CT expansive Osteolyse, die mit einer oder gelegentlich mehreren Zahnwurzeln assoziiert ist. In späteren Stadien wird die Osteolyse dann von der Zahnwurzel her durch ein fast homogenes, zunehmend strahlendichteres Material ausgefüllt, bis nur noch ein schmaler Aufhellungssaum um die Läsion erhalten bleibt (- Abb. 3 a), der dem abgehobenen periodontalen Ligament und der proliferierenden peripheren Komponente des Tumors entspricht. Außerdem kommt es $\mathrm{zu}$ Wurzelresorptionen und einer Obliteration des Periodontalspalts [25].

Makroskopisch präsentiert sich der Tumor als eine abgerundete, knotige, harte Masse, die die Wurzeln eines oder mehrerer Zähne umgibt. Daran schließt sich ein schmaler bindegewebiger Saum an.

Histologisch besteht der Tumor aus flächenartig angeordneten Ablagerungen einer fast zellfreien Hartsubstanz, die vorwiegend zentral deutlich erkennbare pseudopagetoide Kittlinenmuster zeigt. Polarisationsoptisch ist die Hartsubs$\tan z$ faserförmig strukturiert. Die Ablagerungen können größere, zusammenhängende Flächen bilden. Sie werden von einem mäßig zelldichten, gut vaskularisierten Stroma umgeben, das Riesenzellen

\title{
Hier steht eine Anzeige.
}

\author{
Springer
}


enthalten kann. Peripher finden sich radiär angeordnete, überwiegend azelluläre Zementsäulen, die von kubischen Zementoblasten umgeben werden (• Abb. $\mathbf{3}$ b, c). Die differenzialdiagnostische Abgrenzung gegen das Osteoblastom kann histologisch schwierig werden. Dieser Tumor zeigt jedoch keine Fusion mit einer Zahnwurzel, sodass dieser radiologische und morphologische Befund die Unterscheidung ermöglicht. Die Auffassung, die in einigen Monographien über Knochentumoren geäußert wird, dass das „Zementoblastom ein Osteoblastom im Kiefer" sei $[8,24]$, wird den hierzu vorliegenden komplexen Befunden sicher nicht gerecht $[3,27]$. Eine differenzialdiagnostisch $\mathrm{zu}$ erwägende, ebenfalls mit einer Zahnwurzel (meist erster Prämolar) assoziierte Läsion ist die nichtneoplastische Hyperzementose. Sie ist klinisch stumm, weist keine Wachstumstendenz auf und zeigt im Gegensatz zum ZB nur Zementablagerungen, die von einem zellarmen Stroma umgeben werden. Ein Osteosarkom weist ein aggressiveres Röntgenbild sowie keine Fusion mit der Zahnwurzel auf und zeigt histologisch atypische Zellen, deren Atypiegrad manchmal jedoch diskret sein kann.

Das ZB ist zwar gutartig, besonders größere Läsionen zeigen jedoch eine hohe Rezidivneigung (etwa in $20 \%$ der Fälle), wobei es zu lokalen Destruktionen kommen kann. Fast alle Rezidive treten innerhalb von 2 Jahren auf. Therapeutisch wird deshalb empfohlen, Zementoblastome zusammen mit dem betroffenen Zahn und dem unmittelbar angrenzenden Knochen (Ausfräsen) zu entfernen [4].

\section{Fazit für die Praxis}

Bis auf das odontogene Myxom, dem vierthäufigsten odontogenen Tumor, sind die übrigen ektomesenchymalen odontogenen Tumoren (odontogenes Fibrom und Zementoblastom) sehr selten. Während sich die Diagnose des Zementoblastoms aus seiner engen Verbindung mit einer Zahnwurzel ergibt, besteht bei der Diagnose eines odontogenen Fibroms und Myxoms die Gefahr, normale Zahnstrukturen wie Zahnsäckchen bzw. Zahnfollikel und Zahnpapille mit einem odontogenen Fibrom oder Myxom zu verwechseln. Diese Verwechslung kann ernste Folgen haben, da bei einem Myxom unter Umständen eine primäre, ausgedehnte Resektion vorgenommen wird. Hier hilft im Wesentlichen die Beachtung des Alters (selten bei Kindern) und des Röntgenbilds, das im Fall von Normalstrukturen immer einen retinierten Zahn umgeben von einer gut begrenzten Osteolyse zeigt. Außerdem sind oft an der Oberfläche des vermeintlichen „Myxoms" Reste des Schmelzepithels oder Dentinfragmente nachweisbar. In Zweifelsfällen - und besonders bei jungen $\mathrm{Pa}$ tienten in Zusammenhang mit Weisheitszahnextraktionen - ist das Einholen einer Zweitmeinung ratsam.

\section{Korrespondenzadresse}

Prof. Dr. G. Jundt

Institut für Pathologie, DÖSAK-Zentralregister am Knochentumor-Referenzzentrum, Universitätsspital Basel

Schoenbeinstraße 40, 4031 Basel, Schweiz gernot.jundt@unibas.ch

Interessenkonflikt. Der korrespondierende Autor gibt an, dass kein Interessenkonflikt besteht.

\section{Literatur}

1. Araki M, Kameoka S, Mastumoto N et al. (2007) Usefulness of cone beam computed tomography for odontogenic myxoma. Dentomaxillofac Radiol 36: $423-427$

2. Barnes L, Eveson JW, Reichart P et al. (eds) (2005) Pathology and genetics of head and neck tumours. IARC Press, Lyon

3. Bosshardt DD (2005) Are cementoblasts a subpopulation of osteoblasts or a unique phenotype? J Dent Res 84: 390-406

4. Brannon RB, Fowler CB, Carpenter WM et al. (2002) Cementoblastoma: An innocuous neoplasm? A clinicopathologic study of 44 cases and review of the literature with special emphasis on recurrence. Oral Surg Oral Med Oral Pathol Oral Radiol Endod 93: $311-320$

5. Buchner A, Odell EW (2005) Odontogenic myxoma/myxofibroma. In: Barnes L, Eveson J, Reichart P, Sidransky D (eds) Pathology and genetics of head and neck tumours. IARC Press, Lyon, pp 316-317

6. Chuchurru JA, Luberti R, Cornicelli JC et al. (1985) Myxoma of the mandible with unusual radiographic appearance. J Oral Maxillofac Surg 43: 987990

7. Daniels JSM (2004) Central odontogenic fibroma of mandible: a case report and review of the literature. Oral Surg Oral Med Oral Pathol Oral Radiol Endod 98: 295-300

8. Dorfman HD, Czerniak B (1998) Bone tumors. Mosby, St. Louis

9. Fujita S, Hideshima K, Ikeda T (2006) Nestin expression in odontoblasts and odontogenic ectomesenchymal tissue of odontogenic tumours. J Clin Pathol 59: 240-245
10. Gardner DG (1996) Central odontogenic fibroma current concepts. J Oral Pathol Med 25: 556-561

11. Gardner DG (1980) The central odontogenic fibroma: an attempt at clarification. Oral Surg Oral Med Oral Pathol 50: 425-432

12. Gardner DG (1982) The peripheral odontogenic fibroma: An attempt at clarification. Oral Surg Oral Med Oral Pathol 54: 40-48

13. Jing W, Xuan M, Lin Y et al. (2007) Odontogenic tumours: a retrospective study of 1642 cases in a Chinese population. Int J Oral Maxillofac Surg 36: 20-25

14. Keszler A, Dominguez FV, Giannunzio G (1995) Myxoma in childhood: an analysis of 10 cases. J Oral Maxillofac Surg 53: 518-521

15. Kim J, Ellis GL (1993) Dental follicular tissue: misinterpretation as odontogenic tumors. J Oral Maxillofac Surg 51: 762-767

16. Kramer IRH, Pindborg JJ, Shear M (1992) Histological typing of odontogenic tumors. Springer, Berlin Heidelberg New York Tokio

17. Lamberg MA, Calonius BPE, Mäkinen JEA et al. (1984) A case of malignant myxoma (myxosarcoma) of the maxilla. Scand J Dent Res 92: 352-357

18. Lendahl U, Zimmerman LB, McKay RDG (1990) CNS stem cells express a new class of intermediate filament protein. Cell 60: 585-595

19. Pahl S, Henn W, Binger T et al. (2000) Malignant odontogenic myxoma of the maxilla: case with cytogenetic confirmation. J Laryngol Otol 114: 533535

20. Philipsen HP, Reichart PA, Sciubba JJ et al. (2005) Odontogenic fibroma. In: Barnes L, Eveson J, Reichart P, Sidransky D (eds) Pathology and genetics of head and neck tumours. IARC Press, Lyon, p 315

21. Reichart PA, Philipsen HP (2004) Odontogenic tumors and allied lesions. Quintessence Publishing Ca Ltd., London

22. Reichart PA, Philipsen HP, Moegelin A et al. (2006) Central odontogenic fibroma, granular cell variant. Oral Oncol Extra 42: 5-9

23. Suarez PA, Batsakis JG, El-Naggar AK (1996) Don't confuse dental soft tissues with odontogenic tumors. Ann Otol Rhinol Laryngol 105: 490-494

24. Unni KK, Inwards CY, Bridge JA et al. (2005) Tumors of the bones and joints. ARP Press, Silver Springs

25. Waal I van der (2005) Cementoblastoma. In: Barnes L, Eveson J, Reichart P, Sidransky D (eds) Pathology and genetics of head and neck tumours. IARC Press, Lyon, p 318

26. Wiese C, Rolletschek A, Kania G et al. (2004) Nestin expression - a property of multi-lineage progenitor cells? Cell Mol Life Sci 61: 2510-2522

27. Zeichner-David M (2006) Regeneration of periodontal tissues: cementogenesis revisited. Periodontology 2000 41: 196-217 